J. Lake Sci.(湖泊科学), 2009, 21(6): 775-781

http://www.jlakes.org. E-mail: jlakes@niglas.ac.cn

(c)2009 by Journal of Lake Sciences

\title{
太湖梅梁湾水土界面反硝化和厌氧氨氧化*
}

\author{
徐 徽 ${ }^{1}$, 张 路 $^{2 * *}$, 商景阁 ${ }^{2}$, 代静玉 ${ }^{1}$, 范成新 ${ }^{2}$ \\ (1: 南京农业大学资源与环境学院, 南京 210093) \\ (2: 中国科学院南京地理与湖泊研究所, 南京 210008)
}

摘 要: 运用无扰动芯样实验室内流动培养、稳定同位素示踪、同位素气态产物测定及同位素配对技术, 对太湖梅梁湾北部 到南部的 4 个梯度样点的水土界面反硝化和厌氧氨氧化速率进行研究. 结果表明, 梅梁湾内及湾外开敞湖区 4 个样点的水土 界面反硝化脱氮速率为 $(46.36 \pm 13.26)-(16.34 \pm 22.74) \mu \mathrm{mol} /\left(\mathrm{m}^{2} \cdot \mathrm{h}\right)$, 厌氧氨氧化脱氮速率为 $(7.50 \pm 2.21)-(2.05 \pm 2.90) \mu \mathrm{mol} /\left(\mathrm{m}^{2} \cdot \mathrm{h}\right)$. 梅梁湾北部河口区水土界面总脱氮能力明显高于梅梁湾南部及开敞湖区. 通过对脱氮过程的进一步研究发现, 北部脱氮过程 主要以上覆水硝酸盐为底物的非耦合反硝化过程 $\left(D_{\mathrm{w}}\right)$ 为优势过程，而梅梁湾外开敞湖区则以沉积物硝化过程耦合控制的反硝 化 $\left(D_{\mathrm{n}}\right)$ 为主. 影响 $D_{\mathrm{n}} 、 D_{\mathrm{w}}$ 在反硝化中比重的主要因素是沉积物溶氧侵蚀深度和上覆水 $\mathrm{NO}_{3}$ 浓度的差异; 梅梁湾戻氧氨氧化脱 氮比例占总脱氮比例为 $12 \%-14 \%$, 湾外开敞湖区则占 $11 \%$, 影响其比例差异的主要因子是反硝化强度的大小及其反硝化中间 产物一一亚硝酸盐含量的差异.

关键词: 太湖; 沉积物; 反硝化; 厌氧氨氧化

\section{Denitrification and anammox on the sediment-water interface in the Meiliang Bay of Lake Taihu}

\author{
XU Hui ${ }^{1}$, ZHANG Lu ${ }^{2}$, SHANG Jingge ${ }^{2}$, DAI Jingyu ${ }^{1} \&$ FAN Chengxin ${ }^{2}$ \\ (1: College of Resource and Enviromental Sciences, Nanjing Agricultural University, Nanjing 210093, P.R.China) \\ (2: Nanjing Institute of Geography and Limnology, Chinese Academy of Sciences, Nanjing 210008, P.R.China)
}

\begin{abstract}
Undisturbed sediment cores from four sites in Meiliang Bay were collected from north to south of Lake Taihu, then incubated by flow-through technique in the laboratory. The denitrification and anaerobic ammonium oxidation (anammox) rates were measured by stable isotope tracing technique, isotope nitrogen product measurement and isotope pairing technique. The results showed that denitrification and anammox rate were from $46.36 \pm 13.26$ to $16.34 \pm 22.74 \mu \mathrm{mol} /\left(\mathrm{m}^{2} \cdot \mathrm{h}\right)$ in the Meiliang Bay and from $7.50 \pm 2.21$ to $2.05 \pm 2.90 \mu \mathrm{mol} /\left(\mathrm{m}^{2} \cdot \mathrm{h}\right)$ on the open lake areas out of the bay, respectively. Denitrification rates were significantly higher in the north of the Meiliang Bay than in the south and open lake areas. The dominated nitrogen removal process was uncoupled nitrification-denitrification $\left(D_{\mathrm{w}}\right)$ which utilizing nitrate in overlying water as substrate in north Meiliang Bay, and coupled denitrification $\left(D_{\mathrm{n}}\right)$ was dominate process in the open lake area. The concentration of $\mathrm{NO}_{3}^{-}$and $\mathrm{O}_{2}$ penetration depths were main factors affecting the distribution of $D_{\mathrm{n}}$ and $D_{\mathrm{w}}$. The anammox proportion of the total nitrogen removal ratio was $12 \%-14 \%$ in the Meiliang Bay, and $11 \%$ out of the bay, which was correlated with the denitification rate and influenced by the intermediate product (nitrite) of denitrification.
\end{abstract}

Keywords: Lake Taihu; sediment; denitrification; anammox

氮是湖泊生态系统中重要的营养限制因子, 其含量的升高势必会影响到水生生态系统的结构和水生

* 国家自然科学重点基金项目(40730528)和国家自然科学基金项目(40501064)联合资助. 2009-03-04 收稿; 2009-05-20 收 修改稿. 徐徽, 女, 1983 年生, 硕士; E-mail: xh831030@sohu.com.

** 通讯作者; E-mail: luzhang@niglas.ac.cn. 
生物的组成. 因此, 氮元素的水平直接影响着内陆湖泊水环境的安全, 很多湖泊都出现了很高的氮负荷, 如太湖自 20 世纪 70 年代末就开始出现严重的氮污染, 由此可见对脱氮的研究十分重要. 近年来, 随着脱 氮研究的发展, 对其机制提出了多种假设, 如耦合硝化-反硝化脱氮(Coupled Nitrification-denitrification)、非 耦合硝化一反硝化脱氮(Uncoupled Nitrification-denitrification)和厌氧氨氧化过程(Anaerobic Ammonium Oxidation, Anammox). 耦合硝化一反硝化脱氮过程所需的硝酸盐主要来自沉积物中的氨氮经过硝化作 用生成, 而非耦合硝化一反硝化脱氮过程所需的硝酸盐主要来自上覆水中, 厌氧氨氧化是指氨氮在 缺氧的条件下通过硝态氮或亚硝态氮氧化生成氮气 $\left(\mathrm{NH}_{4}{ }^{+}+\mathrm{NO}_{2}{ }^{-} \rightarrow \mathrm{N}_{2}+2 \mathrm{H}_{2} \mathrm{O}\right)$ 的过程, 氨盐和硝酸盐或 亚硝酸盐可以同步去除. 国外对厌氧氨氧化过程在海洋中的研究已经十分成熟 ${ }^{[1-5]}$, 但在湖泊中的研 究报道较少 ${ }^{[6]}$.

由于氮气的本底值极高, 因此脱氮过程的研究进展一定程度上取决于氮气测定手段的改进. 常见的 几种研究方法有: 质量平衡法(Mass-balance Approach)、乙炔抑制法(Acetylene Inhibition Technique)、硝态 氮损耗法(Disappearance of Nitrate)、孔隙水的硝态氮进入沉积物的释放速率、含有 ${ }^{15} \mathrm{~N}$ 的硝态氮稀释法 (Nitrate Dilution)、 $\mathrm{N}_{2}$ 产量法(Direct measurement of $\mathrm{N}_{2}$ production)和同位素配对技术(Isotope Pairing Technique $)^{[7]}$ 等. 质量平衡法是根据氮的输人与输出量估算反硝化速率, 这在大多数情况下很难取得准确 的输人输出量, 结果可信度低 ${ }^{[8]}$. 乙炔抑制法利用一定浓度的乙炔抑制剂使反硝化过程停留在 $\mathrm{N}_{2} \mathrm{O}$ 阶 段，根据 $\mathrm{N}_{2} \mathrm{O}$ 的产量估算反硝化速率. 这是最简单的方法但也有其缺点：(1)乙炔抑制了耦合硝化一反硝 化过程 ${ }^{[9]}$, (2) 在硝态氮浓度很低或硫化物存在时会影响 $\mathrm{N}_{2} \mathrm{O}$ 还原酶活性 ${ }^{[1]},(3) \mathrm{N}_{2} \mathrm{O}$ 扩散到沉积物深层会还 原成 $\mathrm{N}_{2}{ }^{[10]}$ 等, 这些都会导致低估反硝化速率. 硝态氮的损耗并非只有反硝化过程, 它还可以还原为氨氮, 所以测定硝态氮损耗的方法会高估反硝化速率 ${ }^{[11-12]}$. 计算孔隙水的硝态氮进人沉积物的释放速率法与测 定硝态氮损耗法存在同样的问题. ${ }^{15} \mathrm{~N}$ 标记的硝态氮稀释法也忽视了耦合硝化一反硝化过程和生物同化作 用. 直接测定 $\mathrm{N}_{2}$ 产量法则由于 $\mathrm{N}_{2}$ 的本底值很高, 需要非常高精度的仪器, 且无法区分不同的反硝化过程. 同位素配对技术是将 ${ }^{15} \mathrm{NO}_{3}$ 指示剂加人到沉积物上覆水中, ${ }^{15} \mathrm{NO}_{3}$ 指示剂与上覆水中原有的 ${ }^{14} \mathrm{NO}_{3}$ 混合并 进人到表层沉积物中. 这种技术的优点在于它可以同时揭示耦合与非耦合硝化一反硝化反应 ${ }^{[13]}$ 的比例和 速率. 本论文结合同位素配对技术和膜接口质谱仪(Membrance Inlet Mass Spectrometry, MIMS)测定同位 素反硝化产物 ${ }^{[14]}$ 的方法来测定反硝化速率, 并区分不同的反硝化途径. 即通过膜接口装置将溶解性气体 转化为气体形态, 直接进入质谱在线测定水样中氮气, 利用测定出的氮气同位素产物与同位素配对法计 算出耦合和非塊合的反硝化以及Anammox脱氮速率，具有测定速度快、所需样品量少、测定灵敏度和精 度高的特点.

\section{1 材料与方法}

\section{1 样点布设}

太湖是一个富营养化的大型浅水湖泊, 受北部梁溪河、问江等河道输人和无锡市的影响, 位于太湖 北部的梅梁湾属于富营养化程度较高的藻型湖区, 由于人湖污染主要来自梅梁湾北部, 并向南逐渐稀释 扩散, 水体及沉积物存在自然梯度, 其中北部因接近人湖河口, 其沉积物氮磷浓度高于梅梁湾开敞湖区. 因此, 选择梅梁湾内 3 个样点及梅梁湾外 1 个样点作为对比研究(图 1), 并便于与已有研究比较 ${ }^{[15-16]}$. 其 中梅梁湾内由北向南设置 $0^{\#} 、 1^{\#} 、 3^{\#}$ 点, 湾外设置 $7^{\#}$ 点. 样点具体经纬度见表 1.

\section{2 样品采集}

2008 年 12 月在各样点采集样品, 用有机玻璃柱状采样器(内径为 $77 \mathrm{~mm}$ )采集每个样点的 3 个柱 样并现场切表层 $2 \mathrm{~cm}$, 放人自封袋混合保存, 小心带回实验室进行样品分析. 另采集 4 根未扰动柱样, 柱样深度不低于 $10 \mathrm{~cm}$, 柱样上端保留原上覆水样, 两端用橡皮塞塞紧后垂直放置, 尽量无扰动的运 回实验室; 同步采集上覆水 25L，用多参数水质测定仪(YSI 6600V2，美国)测定现场上覆水基本理化 参数(表 1). 

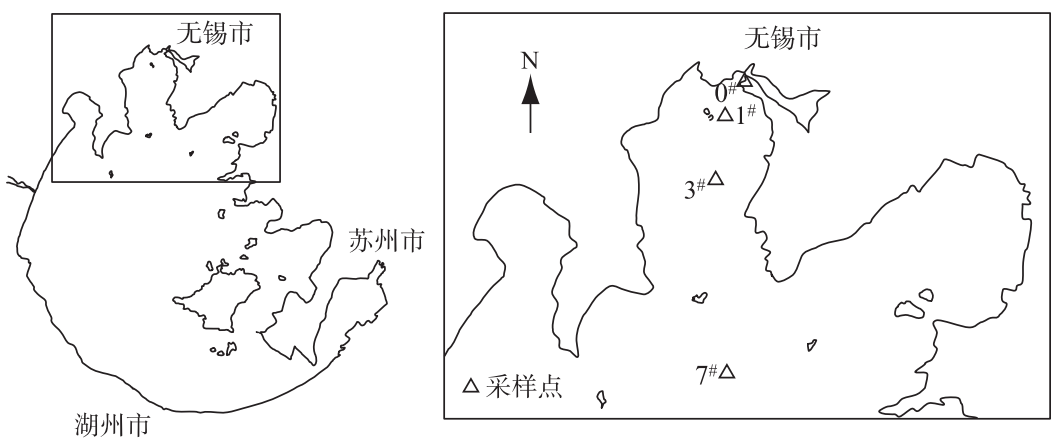

图 1 太湖梅梁湾采样点分布

Fig.1 Location of sampling sites in Meiliang Bay of Lake Taihu

表 1 采样点水体基本理化参数

Tab.1 General character of Meiliang Bay measured by YSI

\begin{tabular}{cccccccc}
\hline 采样点 & 经纬度 & \multicolumn{2}{c}{ 水深 $(\mathrm{m})$ 水温 $\left({ }^{\circ} \mathrm{C}\right)$} & 溶解氧 $(\mathrm{mg} / \mathrm{L})$ & 叶绿素 $(\mu \mathrm{g} / \mathrm{L})$ & 矿化度 $(\mathrm{mg} / \mathrm{L})$ & $\mathrm{ORP}(\mathrm{mV})$ \\
\hline $0^{\#}$ & $120^{\circ} 12^{\prime} 47^{\prime \prime}, 31^{\circ} 32^{\prime} 08^{\prime \prime}$ & 2.5 & 9.0 & 12.14 & 9.1 & 0.379 & 91.6 \\
$1^{\#}$ & $120^{\circ} 10^{\prime} 47^{\prime \prime}, 33^{\circ} 30^{\prime} 46^{\prime \prime}$ & 2.0 & 8.3 & 12.30 & 5.1 & 0.323 & 99.1 \\
$3^{\#}$ & $120^{\circ} 11^{\prime} 42^{\prime \prime}, 31^{\circ} 29^{\prime} 10^{\prime \prime}$ & 1.8 & 7.7 & 12.02 & 10.0 & 0.358 & 95.2 \\
$7^{\#}$ & $120^{\circ} 11^{\prime} 42^{\prime \prime}, 30^{\circ} 21^{\prime \prime 38 "}$ & 1.4 & 7.6 & 12.14 & 2.5 & 0.333 & 102.4 \\
\hline
\end{tabular}

\section{3 室内培养实验}

实验室内垂直放置采集的柱样，打开上端橡皮塞，用注射器沿着管壁尽量无扰动的注满上覆水，放 置在与现场水温一致的水浴锅中过夜，去除运输过程中短暂密封造成的水体溶解氧下降等因素. 用溶解 氧微电极系统(Presens, 德国)测定不同样点的溶解氧侵蚀深度. 该系统由测定主机、光线溶解氧微电极、 高分辨率三维控制器三部分组成. 溶解氧微电极为针式, 针尖直径可细至 $40 \mu \mathrm{m}$, 反应时间 $1 \mathrm{~s}$ 以内, 测定 时不消耗氧. 三维控制器垂直分辨率理论上可达到 $20 \mu \mathrm{m}$. 测完溶解氧侵蚀深度后, 对上述柱样进行无顶 空流动培养. 进水管距表层泥约 $1 \mathrm{~cm}$, 进水管低于出水管, 通过蠕动原将进水以恒定的流速 $(1 \mathrm{ml} / \mathrm{min})$ 尿 人柱样中, 使流动培养的进出水循环形成垂直及水平方向的混合水流, 使上覆水充分混合, 并通过持续 流动保持上覆水中离子浓度稳定. 柱样用与现场温度一致的恒温水浴培养 $\left(7^{\circ} \mathrm{C}\right)$, 由于样点透明度均小于 水深, 因此流动培养在避光条件下进行. 流动培养的柱样作两种处理, 其中两根柱样的进水为原位采集 的湖水(对照实验), 另两根柱样的进水中加人 $\mathrm{Na}^{15} \mathrm{NO}_{3}$ 并使最终浓度达到 $100 \mu \mathrm{mol} / \mathrm{L}$ (添加同位素实验). 表层沉积物样烘干碾磨过篮, 用过硫酸钾法测定总氮、总磷, 烧失法测定有机物含量(LOI) (表 2).

表 2 上覆水及沉积物化学性质

Tab.2 Chemical properties of water and sediments from Meiliang Bay

\begin{tabular}{|c|c|c|c|c|c|}
\hline \multirow{2}{*}{ 采样点 } & \multicolumn{2}{|c|}{ 上覆水 } & \multicolumn{3}{|c|}{ 沉积物 } \\
\hline & $\mathrm{NO}_{3}{ }^{-}(\mathrm{mg} / \mathrm{L})$ & $\mathrm{NH}_{4}^{+}(\mathrm{mg} / \mathrm{L})$ & 总氮(\%) & 总磷(\%) & LOI (\%) \\
\hline $0^{\#}$ & 0.78 & 0.47 & 0.208 & 0.0665 & 4.69 \\
\hline $1^{\#}$ & 0.41 & 0.04 & 0.221 & 0.0485 & 5.49 \\
\hline $3^{\#}$ & 0.57 & 0.08 & 0.208 & 0.0427 & 5.07 \\
\hline $7^{\#}$ & 0.56 & 0.10 & 0.152 & 0.0326 & 4.35 \\
\hline
\end{tabular}




\section{4 测定方法}

用注射器小心采集进水样品, 在无气泡产生情况下充分溢流并装满细长的溶解性气体采集管, 出水 样品直接溢流收集, 收集的样品立即用膜接口质谱仪测定溶液中可溶性气体的量, 主要测定指标为 ${ }^{28} \mathrm{~N}_{2}$, ${ }^{29} \mathrm{~N}_{2},{ }^{30} \mathrm{~N}_{2}$ 和氧气. 另收集 $12 \mathrm{ml}$ 进、出水样品, 经 $0.45 \mu \mathrm{m}$ 醋酸纤维滤膜过滤后, 用流动注射分析仪 (Skalar-SA1000)测定 $\mathrm{NO}_{3}{ }^{-}$和 $\mathrm{NH}_{4}{ }^{+}$浓度.

\section{5 计算方法}

沉积物中反硝化作用发生所需的 $\mathrm{NO}_{3}$-可能来自上覆水 $\left(D_{\mathrm{w}}\right)$ 或来自沉积物中的硝化作用 $\left(D_{\mathrm{n}}\right)$. 这两种 过程都可以通过同位素配对技术来测定. 将 ${ }^{15} \mathrm{NO}_{3}$ 加人到上覆水中, 加人的 ${ }^{15} \mathrm{NO}_{3}$ 与上覆水和沉积物表 层中原有的 ${ }^{14} \mathrm{NO}_{3}$ 混合. 由这些硝酸盐产生的反硝化产物— $\mathrm{N}_{2}$ 的分子量有 $28,29,30$.

$\mathrm{N}_{2}$ 的释放速率 $\left(r_{29}, r_{30}\right)^{[17]}$ :

$$
r_{n}=\left(C_{n}-C_{n, o}\right) V / S \times 60 \times 24
$$

式中, $C_{n} 、 C_{n, o}$ 为第 $n$ 次取样时出水及进水中氮气含量 $(\mathrm{mg} / \mathrm{L}) ; V$ 为蠕动葲流速 $(\mathrm{ml} / \mathrm{min}) ; S$ 为柱样中水-沉积 物界面面积 $\left(\mathrm{m}^{2}\right) ; 60$, 24为时间换算因子.

通过生成的 ${ }^{29} \mathrm{~N}_{2},{ }^{30} \mathrm{~N}_{2}$, 计算利用 ${ }^{15} \mathrm{NO}_{3}{ }^{-}$而发生的反硝化量 $D_{15}{ }^{[13]}$ :

利用未标记的 ${ }^{14} \mathrm{NO}_{3}{ }^{-}$产生的反硝化量 $D_{14}{ }^{[12]}$ :

$$
D_{15}=r_{29}+2 \times r_{30}
$$

沉积物中总的反硝化量 $D_{\mathrm{tot}}{ }^{[13]}$ :

$$
D_{14}=D_{15} r_{29} /\left(2 \times r_{30}\right)
$$

通过以上的结果计算 $D_{\mathrm{n}}, D_{\mathrm{w}}$ 速率 ${ }^{[13]}$ :

$$
D_{\mathrm{tot}}=D_{15}+D_{14}
$$

$$
\begin{gathered}
D_{\mathrm{n}}=D_{\mathrm{tot}}-D_{15} / \varepsilon \\
D_{\mathrm{w}}=D_{15} / \varepsilon \times(1-\varepsilon) \\
\varepsilon=\left(\left[\mathrm{NO}_{3}\right]_{\mathrm{a}}-\left[\mathrm{NO}_{3}^{-}\right]_{\mathrm{b}}\right) /\left[\mathrm{NO}_{3}\right]_{\mathrm{a}}
\end{gathered}
$$

式中, $\varepsilon$ 代表培养试验中 ${ }^{15} \mathrm{NO}_{3}$ 的丰度, 下标a和b表示添加同位素之后和之前.

计算厌氧氨氧化(Anammox)速率 ${ }^{[2]}$ :

$$
\begin{gathered}
A_{\mathrm{tot}}=\left[r_{29}+2 \times\left(1-\varepsilon^{-1}\right) r_{30}\right] \varepsilon \\
A_{28}=A_{\mathrm{tot}}(1-\varepsilon)
\end{gathered}
$$

式中, $A_{\mathrm{tot}}$ 代表Anammox过程生成 ${ }^{28} \mathrm{~N}_{2},{ }^{29} \mathrm{~N}_{2}$ 的总通量, $A_{28}$ 代表Anammox过程生成 ${ }^{28} \mathrm{~N}_{2}$ 的通量.

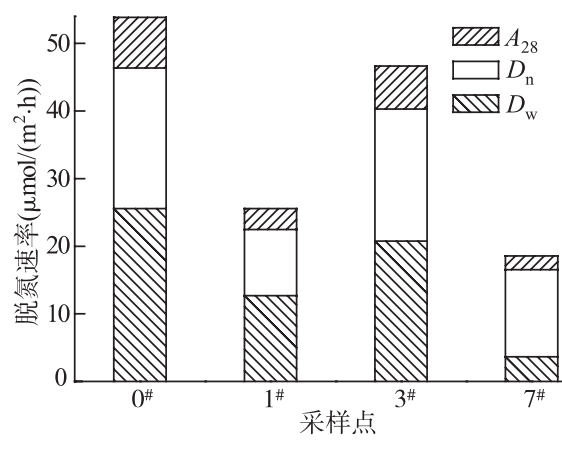

图2 梅梁湾不同样点的脱氮速率

Fig.2 The fluxes of nitrogen removing in different sampling sites of Meiliang Bay $\mathrm{NO}_{3}$ - 浓度显著相关 $(P<0.05)$. 即上覆水 $\mathrm{NO}_{3}^{-}$浓度越高, 提供给反硝化的底物也越多, 相应的反硝化速 率越高.

\section{2 结果与讨论}

\section{1 梅梁湾水土界面反硝化的空间变化}

通过MIMS 测定样品中氮气及其同位素组分, 并计算各 点的脱氮速率. 总反硝化速率 $\left(D_{14}=D_{\mathrm{n}}+D_{\mathrm{w}}\right)$ 在 $0^{\#}$ 点最强, 速率 为 $46.36 \pm 13.26 \mu \mathrm{mol} /\left(\mathrm{m}^{2} \cdot \mathrm{h}\right)$ (平均值土标准差, 下同), $1^{\#}$ 反硝化 速率为 $22.45 \pm 20.52 \mu \mathrm{mol} /\left(\mathrm{m}^{2} \cdot \mathrm{h}\right), 3^{\#}$ 为 $40.30 \pm 29.71 \mu \mathrm{mol} /\left(\mathrm{m}^{2} \cdot \mathrm{h}\right)$, $7^{\#}$ 最弱为 $16.34 \pm 22.74 \mu \mathrm{mol} /\left(\mathrm{m}^{2} \cdot \mathrm{h}\right)$, 总体表现为梅梁湾北部反 硝化速率( 3 个点的反硝化速率均值为 $36.37 \mu \mathrm{mol} /\left(\mathrm{m}^{2} \cdot \mathrm{h}\right)$ ) 高于 湾外开敞湖区(图2). 梅梁湾北部上覆水 $\mathrm{NO}_{3}$-浓度表现为北 部高南部低(表2), 但 $3^{\#}$ 情况略有特殊, 与该点沉积物溶解 氧侵人深度、沉积物耗氧量等物化性质有关(详见2.2节), 且与上覆水硝酸盐浓度有紧密联系. 通过反硝化速率与上 覆水硝酸盐含量的回归分析发现，反硝化速率与上覆水 


\section{2 耦合与非耦合反硝化过程}

梅梁湾内 3 个样点和湾外开敞湖区反硝化脱氮的方 式不同(图2). 梅梁湾内样点耦合 $\left(D_{\mathrm{n}}\right)$ 、非耦合反硝化 $\left(D_{\mathrm{w}}\right)$ 速率平均值分别为 $19.68 \pm 6.51 \mu \mathrm{mol} /\left(\mathrm{m}^{2} \cdot \mathrm{h}\right)\left(0^{\# 、} \mathrm{1}^{\#}\right.$ 及 $3^{\#}$ 平均 值, 下同)和 $16.69 \pm 6.04 \mu \mathrm{mol} /\left(\mathrm{m}^{2} \cdot \mathrm{h}\right)$, 湾外开敞湖区则为 $3.65 \pm 4.29 \mu \mathrm{mol} /\left(\mathrm{m}^{2} \cdot \mathrm{h}\right)\left(7^{\#}\right.$, 下同 $)$ 和 $12.87 \pm 18.20 \mu \mathrm{mol} /\left(\mathrm{m}^{2} \cdot \mathrm{h}\right)$.

一般认为, 溶解氧消失的位置是反硝化层的上界面, 就是说溶解氧侵人深度等于反硝化层深度, 也等于沉积 物硝化层厚度, 因此对于耦合硝化-反硝化 $\left(D_{\mathrm{n}}\right)$ 来说, 氧 气侵蚀深度越大越有利于沉积物硝化层的硝化作用 ${ }^{[7]}$, 并进一步提高沉积物的耦合硝化-反硝化作用. 而氧气侵 蚀深度小, 沉积物上部的好氧层厚度薄, 则有利于水体 中的硝酸盐向反硝化好氧层下的反硝化层扩散, 并作为 反硝化底物形成非耦合反硝化 ${ }^{[7]}$. 太湖梅梁湾及湾外开

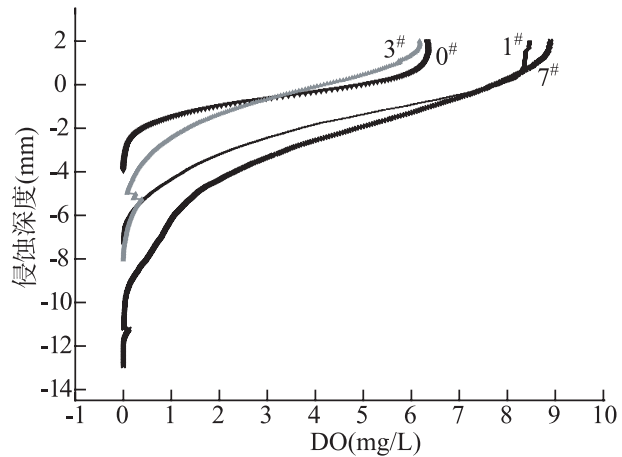

图3 梅梁湾不同样点沉积物溶解氧侵蚀深度

Fig.3 The penetration depths of DO in different sampling sites of Meiliang Bay

敞湖区不同采样点沉积物的氧气的垂向剖面差异明显(图3), 其侵蚀深度范围在3.9-13mm, 大体表现为 梅梁湾内氧气侵人深度要小于开敞湖区, 王建军等在类似的研究中也发现了同样的规律 ${ }^{[18]}$. 经回归分析 发现氧气侵人深度与非耦合反硝化速率呈负相关 $(r=-0.812)$, 这与Nielsen ${ }^{[7]}$ 在同位素配对法计算中提出 的理论依据一致.

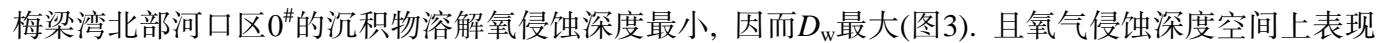
为湾内浅开敞湖区深，因此 $D_{\mathrm{n}}$ 占总脱氮速率的比值也表现为开敞湖区高 $69.96 \%$ )而湾内低(3个点平均值 $39.53 \%)$, 而 $D_{\mathrm{w}}$ 的比值则是湾内 (3个点平均值 $\left.47.21 \%\right)$ 高于开敞湖区 $(19.85 \%)$, 也即梅梁湾北部反硝化所 需的硝酸盐主要来自上覆水中, 南部反硝化所需的硝酸盐主要来自沉积物中氨氮的硝化作用.

非耦合硝化一反硝化 $\left(D_{\mathrm{w}}\right)$ 所需的 $\mathrm{NO}_{3}$ - 来自上覆水的扩散，因此上覆水硝酸盐的浓度及交换速率都可 能会影响反硝化速率 ${ }^{[19]}$. 将二者与 $D_{\mathrm{w}}$ 值做Pearson相关性分析发现, 上覆水 $\mathrm{NO}_{3}{ }^{-}$浓度与 $D_{\mathrm{w}}$ 显著相关 $(P<0.05, r=0.997)$, 而硝酸盐释放速率对 $D_{\mathrm{w}}$ 的影响并不显著 $(P>0.05, r=-0.896)$. 因此, 在 $\mathrm{NO}_{3}{ }^{-}$含量高的梅 梁湾北部地区的 $D_{\mathrm{w}}$ 值最高, 同时也可以知道, 在 $\mathrm{NO}_{3}{ }^{-}$含量高的冬春季节 $D_{\mathrm{w}}$ 值要高于夏秋季. 耦合硝化一 反硝化 $\left(D_{\mathrm{n}}\right)$ 所需的 $\mathrm{NO}_{3}{ }^{-}$来自沉积物间隙水中 $\mathrm{NH}_{4}{ }^{+}$的硝化作用，但是经相关性分析发现间隙水中 $\mathrm{NH}_{4}{ }^{+}$的浓 度并不制约着 $D_{\mathrm{n}}(P>0.05)$, 说明耦合反硝化过程并不仅仅取决于氨氮底物的浓度, 更受沉积物硝化层的 硝化作用强弱制约.

\section{3 厌氧氨氧化的影响因子}

梅梁湾水土界面Anammox $\left(A_{28}\right)$ 的速率由北向南依次为: $7.50 \pm 2.21 \mu \mathrm{mol} /\left(\mathrm{m}^{2} \cdot \mathrm{h}\right), 3.13 \pm 3.13 \mu \mathrm{mol} /\left(\mathrm{m}^{2} \cdot \mathrm{h}\right)$, $6.34 \pm 4.84 \mu \mathrm{mol} /\left(\mathrm{m}^{2} \cdot \mathrm{h}\right), 2.05 \pm 2.90 \mu \mathrm{mol} /\left(\mathrm{m}^{2} \cdot \mathrm{h}\right)$. Anammox过程在整个脱氮过程中所占的比率范围由北向南 依次为 $14 \% 、 12 \% 、 14 \%$ 和 $11 \%$, 均超过 $10 \%$. 许多文献报道海洋中沉积物的厌氧氨氧化脱氮在反硝化过 程中所占的比例最多可超过 $50 \%$ 年, 对海洋氮的去除起到很重要的作用. 但对湖泊而言, 由于沉积物中 往往并不缺乏有机碳, 因此以氨氮为电子供体、硝酸盐为电子受体的Anammox过程与有机碳作为电子供 体的反硝化过程竞争时, 并不能获得优势, 这一点与海洋沉积物低有机碳含量的特点有很大差异 ${ }^{[2]}$. 但 本研究通过同位素示踪的方式至少证实了太湖沉积物中较高的氨氮水平下厌氧氨氧化过程的发生.

Anammox 过程的影响因子很多, 如: 水深、温度、有机质等 ${ }^{[2,5,20]}$. 梅梁湾北部和南部样点在上覆水 硝酸盐浓度和沉积物性质等的差异都很明显, 这些因素都可能会影响反硝化和 Anammox 过程 $^{[3]}$. 根据 Rysgaard 的研究 ${ }^{[3]}$, 海洋中水深、 $\mathrm{NO}_{3}{ }^{-}$浓度及盐度对 Anammox 的影响最显著, 尤其是 $\mathrm{NO}_{3}{ }^{-}$的浓度 $(P<0.0001)$. 然而, 如将本研究中梅梁湾各样点的厌氧氨氧化速率与各参数做 Pearson 相关性分析(表 3$)$, 结果发现对于太湖梅梁湾而言, 上覆水 $\mathrm{NO}_{3}$ 浓度对厌氧氨氧化速率影响并不显著, 反而是反硝化速率对 其影响显著 $(P<0.0001)$, 反硝化速率越高厌氧氨氧化速率也越高. Rysgaard ${ }^{[3]}$ 的研究也表明, 当 Anammox 过程所需的 $\mathrm{NO}_{2}^{-}$由反硝化细菌生成, 那么反硝化速率与 Anammox 速率之间可以产生一定得相关性. 
表3 Anammox与各影响因素的相关关系 ${ }^{1)}$

Tab.3 Pearson correlation coefficients of anammox in Meiliang Bay

\begin{tabular}{cccc}
\hline 相关因素 & $r$ & $P$ & $n$ \\
\hline 水深 & 0.514 & 0.193 & 8 \\
上覆水 $\mathrm{NO}_{3}{ }^{- \text {浓度 }}$ & 0.107 & 0.802 & 8 \\
$\mathrm{NO}_{3}{ }^{-}$交换速率 & -0.404 & 0.321 & 8 \\
$\mathrm{NH}_{4}^{+}$交换速率 & -0.541 & 0.166 & 8 \\
有机质 & 0.058 & 0.942 & 4 \\
反硝化速率 $\left(D_{14}\right)$ & 0.994 & $<0.0001^{* *}$ & 8 \\
\hline
\end{tabular}

1) $r$ : 相关系数; $P$ : 显著因子, **表示显著相关.

\section{4 添加同位素对实验结果的影响}

同位素配对技术的建立基于四点假设 ${ }^{[9]}$ : 一是添加的 ${ }^{15} \mathrm{NO}_{3}{ }^{-}$不会干扰原位的 $\mathrm{NO}_{3}{ }^{-}$的反硝化过程，有研

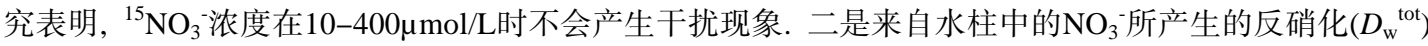
随 $\mathrm{NO}_{3}$-浓度的增高而显现增加. 三是添加的 ${ }^{15} \mathrm{NO}_{3}$-同时标记于水柱与沉积物中. 四是添加 ${ }^{15} \mathrm{NO}_{3}{ }^{-}$后, 在水 土界面会建立一个稳定的 $\mathrm{NO}_{3}$-浓度梯度. 为了验证本添加实验并没有影响原位的反硝化过程, 比较两者 的实验结果(图4). 经单因素方差分析表明, 添加同位素后与不添加同位素(控制组)的计算结果无显著差 异 $(P>0.05)$, 因此用同位素添加示踪的方法获取的反硝化结果能比较真实的反映原位反硝化速率，同时 提供了不同反硝化过程的信息, 有助于深人理解不同反硝化过程对湖泊脱氮过程的贡献.

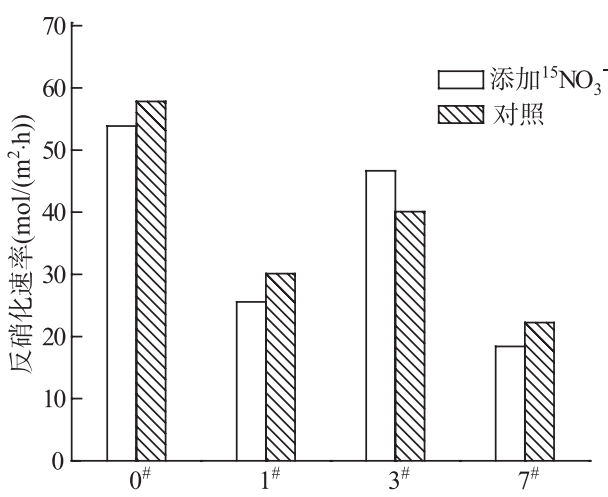

图4 原位培养和添加同位素培养的反硝化速率

Fig.4 The fluxes of denitrification calculated from control and isotope paring incubation

\section{3 结论}

(1)梅梁湾内水土界面的反硝化速率高于湾外开敞湖区，且由于沉积物溶解氧侵蚀深度和上覆水 $\mathrm{NO}_{3}$ 浓度的差异, 导致梅梁湾内以非耦合硝化-反硝化脱氮方式为主, 湾外开敞湖区以耦合硝化-反硝化 脱氮方式为主.

(2)太湖梅梁湾 Anammox 过程占总的脱氮速率的 11\%-14\%, 分析 Anammox 的各个影响因素发现, 梅 梁湾反硝化速率对其影响显著.

(3)通过比较添加与不添加同位素的实验结果表明, 添加同位素示踪的方法获取的反硝化结果能比较 真实的反映原位反硝化速率.

\section{4 参考文献}

[1] Binnerup SJ, Jensen K, Revesbech NP et al. Denitrification, dissmilatory reduction of nitrate to ammonium, and nitrification in 
a bioturbated estuarine sediment as measured with ${ }^{15} \mathrm{~N}$ and microsensor techniques. Appl Environ Microbiol, 1992, 58: $303-313$.

[2] Thamdrup B, Dalsgaard T. Production of $\mathrm{N}_{2}$ through anaerobic ammonium oxidation coupled to nitrate reduction in marine sediments. Appl Environ Microbiol, 2002, 68(3): 1312-1318.

[3] Rysgaard S, Glud RN, Risgaard-Petersen N et al. Denitrification and anammox activity in Arctic marine sediment. Limnol Oceanogr, 2004, 49(5): 1493-1502.

[4] Rao AMF, McCarthy MJ, Gardner WS et al. Respiration and denitrification in permeable continental shelf deposits on the South Atlantic Bight: N2: Ar and isotope pairing measurementsin sediment column experiments. Continental Shelf Research, 2008, 28: 602-613.

[5] Engstron P, Dalsgaard T, Hulth S et al. Anaerobic ammonium oxidation by nitrite (anammox): Implications for $\mathrm{N}_{2}$ production in coastal marine sediments. Geochimica et Cosmochimica Acta, 2005, 69(8): 2057-2065.

[6] Schubert CJ, Durisch KE, Wehrli B et al. Anaerobic ammonium oxidation in a tropical freshwater system (Lake Tanganyika). Environmental Microbiology, 2006, 8(10): 1875-1863.

[7] Nielsen LP. Denitrification in sediment determined from nitrogen isotope pairing. FEMS Microbiol Ecol, 1992, 86: 181-186.

[8] 徐继荣, 王友绍, 孙 松. 海岸带地区的固氮、氨化、硝化和反硝化特征. 生态学报, 2004, 24(12): $2907-2914$.

[9] Lohse L, Kloosterhuis HT, Raaphorst W et al. Denitrification rates as measured by the isotope paring method and by the acetylene inhibition technique in continental shelf sediments of the North Sea. Mar Ecol Prog Ser, 1996, 29: 169-179.

[10] Seizinger SP, Nielsen LP, Caffret J et al. Denitrification measurement in aquatic sediment: a comparision of three methods. Biogeochemistry, 1993, 23: 147-167.

[11] Mengis M, Pernasconi S, Gachter R et al. Nitrogen elimination in two deep eutrophic lakes. Limnol Oceanogr, 1997, 41: 1530-1543.

[12] Rysgaard S, Risgaard-Petersen N, Nielsen LP et al. Nitrification and denitrification in lake and estuarine sediment measured by the ${ }^{15} \mathrm{~N}$ dilution technique and isotope pairing. Appl Environ Microbiol, 1993, 59: 2093-2098.

[13] Steingruber SM, Friedrich J, Gachter R et al. Measurement of Denitrification in Sediments with the ${ }^{15} \mathrm{~N}$ Isotope Pairing Technique. Appl Environ Microbiol, 2001, 67(9): 3771-3778.

[14] McCarthy MJ, Gardner WS. An application of membrane inlet mass spectrometry to measure denitrification in a recirculating mariculture system. Aquaculture, 2003, 218: 341-355.

[15] 范成新, 张 路, 秦伯强等. 太湖沉积物-水界面生源要素迁移机制及其定量化- I. 铵态氮释放速率的空间差异及源-汇 通量. 湖泊科学, 2004, 16(1): 8-16.

[16] McCarthy MJ, Lavrentyev PJ, Yang LY et al. Nitrogen dynamics and microbial food web structure during a summer cyanobacterial bloom in a subtropical, shallow, well-mixed, eutrophic lake (Lake Taihu, China). Hydrobiologia, 2007, 581: 195-207.

[17] Kana TM, Sullivan MB, Cornwell JC et al. Denitrification in estuarine sediments determined by membrane inlet mass spectrometry. Limnol Oceanogr, 1998, 43(2): 334-339.

[18] 王建军. 湖泊水土界面室内模拟系统的建立及其在界面氧气生物地球化学过程研究中的应用[博士论文]. 南京: 中国科 学院南京地理与湖泊研究所, 2008: 31-33

[19] Poulin P, Pelletier E, Saint-Louis R. Seasonal variability of denitrification efficiency in northern salt marshes: An example from the St. Lawrence Estuary. Marine Environmental Research, 2007, 63: 490-505.

[20] Thamdrup B, Dalsgaard T, Jensen MM et al. Anaerobic ammonium oxidation in the oxygen-deficient waters off northern Chile. Limnol Oceanogr, 2006, 51(5): 2145-2156. 